\title{
¿Puede asociarse el sedentarismo con hallazgos clínicos de alarma de enfermedad crónica en adultos jóvenes? Un análisis en el proyecto CHICAMOCHA
} Can Sedentarism be Associated with Alarm Clinical Findings of Chronic Diseases in Young Adults? An Analysis in CHICAMOCHA Project Poderia associar-se o sedentarismo às conclusões clínicas
de alarme de doenças crônicas em adultos jovens?
Análise no projeto CHICAMOCHA

Juan Carlos Villar-Centeno, MD., Esp., MSc., PhD.*

Víctor Mauricio Herrera-Galindo, MD., MSc., PhD.**

Karen Julieth Moreno-Medina, Fist., Esp., ${ }^{* * *}$

\author{
Yeny Zulay Castellanos-Domínguez**** \\ Luz Ximena Martínez-Contreras, MD.Nutri y Diet***** \\ Olga Lucía Cortés, Enf,. Esp., MSc., PhD******
}

\begin{abstract}
Resumen
Introducción: La asociación entre sedentarismo y enfermedades crónicas no transmisibles (ECNT) requiere décadas de exposición. Es posible que esta se manifieste más tempranamente, por algunos hallazgos clínicos en adultos jóvenes. Objetivo: Probar la hipótesis de que en adultos jóvenes el sedentarismo se asocia con algunos signos o síntomas de alarma para el desarrollo posterior de ECNT. Metodología: Usando la evaluación inicial (años 2000-2003) del proyecto CHICAMOCHA, en 1539 donantes de sangre clínicamente saludables con pruebas de tamización negativas (edad media 36, DE 8,3 años, 66\% hombres) se estudió la asociación entre sedentarismo y una
\end{abstract}

serie de hallazgos clínicos. Se definió sedentarismo como reportar actividad física moderada-intensa $\leq 150$ minutos/semana (incluyendo el trabajo). El desenlace primario fue el compuesto de 11 hallazgos (5 síntomas y 6 signos) de alarma encontrados en la valoración médica. La asociación fue estimada usando un modelo regresión logística ajustado por covariables. Resultados: Se encontró que 56.9\% (IC95\% 54.3-59.3) de los participantes eran sedentarios. En el análisis multivariado, el sedentarismo se asoció positivamente con el estado civil soltero y negativamente con estar empleado. No se encontraron asociaciones significativas en el compuesto agregado de 5 síntomas (OR ajustado 1.07, IC95\% 0.90-1.26), 6 signos (OR ajustado 1.01, IC95\% $0.79-1.28$ ). Sin embargo, se observó un gradiente positivo no significativo por el número

\footnotetext{
* Médico, Especialista en Medicina Interna, Magister y Doctor en Epidemiología Clínica; Facultad de Ciencias de la Salud. Grupo de Cardiología Preventiva. Universidad Autónoma de Bucaramanga.

** Médico, Magíster y Doctor en Epidemiología; Facultad de Ciencias de la Salud. Grupo de Cardiología Preventiva. Universidad Autónoma de Bucaramanga.

*** Fisioterapeuta, Especialista en Epidemiología. Departamento de Investigaciones Fundación Cardioinfantil-Instituto de Cardiología.

**** Bacterióloga y Laboratorista Clínico, Especialista en Auditoría de Servicios de Salud, Magíster en Epidemiología; Grupo de Cardiología Preventiva. Universidad Autónoma de Bucaramanga.

***** Médico, Nutricionista y Dietista; Facultad de Ciencias de la Salud. Grupo de Cardiología Preventiva. Universidad Autónoma de Bucaramanga

******Enfermera, Especialista en Docencia Universitaria y Cuidado Crítico del Adulto, Magíster y Doctor en Ciencias Clínicas del Cuidado, Departamento de investigaciones Fundación Cardioinfantil-Instituto de Cardiología.
}

Correspondencia: Juan Carlos Villar Centeno. Calle 157 No. 14 - 55 Cañaveral Floridablanca, Colombia. Universidad Autónoma de Bucaramanga. Teléfono 6436111 extensión 516. Email: jvillar@unab.edu.co 
de hallazgos presentes ( 1 hallazgo OR=0.91, IC95\% 0.61-1.35), 2 hallazgos (OR=1.20, IC95\% 0.84-1.73), 3 o más hallazgos (OR=1.31, IC 95\% 0.91-1.89). Conclusiones: Más de la mitad de la población estudiada se encontró sedentaria. Aunque este factor no se encontró asociado con signos o síntomas individualmente, se identificó un gradiente no significativo con el número de estos hallazgos, posiblemente relacionado con el tiempo de exposición relativamente breve. [Villar JC, Herrera VM, Moreno-Medina KJ, Castellanos-Domínguez YZ, Martínez LX, Cortés OL. ¿Puede asociarse el sedentarismo con hallazgos clínicos de alarma de enfermedad crónica en adultos jóvenes? Un análisis en el proyecto CHICAMOCHA. MedUNAB 2015; $18(1): 42-50]$

Palabras clave: Actividad Motora; Estilo de Vida Sedentario; Factores de Riesgo; Enfermedad Crónica; Prevención Primaria.

\section{Abstract}

Introduction: The association between Sedentary Lifestyle (SL) and Chronic Non-Communicable Diseases (NCD) takes decades of exposure. It is possible to be seen at an early stage in young adults due to some clinical findings. Objective: Test the hypothesis that a sedentary lifestyle in young adults is associated with some signs or symptoms of alarm for the further development of NCD. Methodology: Using the initial evaluation (years 2000-2003) of $\mathrm{CHICAMOCHA}$ project, it was found that 1539 blood donors were healthy with negative screening test results (mean age 36, SD 8.3 years, 66\% male). The association between sedentary lifestyle and a series of clinical findings was studied. Sedentary Lifestyle was defined as moderateintense physical activity of $\leq 150$ minutes/week (including work). The primary outcome was the composite of 11 findings (5 symptoms and 6 signs) found in the medical assessment. We computed multivariate logistic regression models for both individual and pooled outcomes. Results: SL was found in 56.9\% (IC95\% 54.3-59.3) participants. In multivariate analysis SL was positively associated with single marital status and negatively associated with being employed. There were no significant associations between SL and the composite of 5 symptoms (Covariate-adjusted pooled OR $1.07,95 \% \mathrm{Cl} 0.90-1.26$ ), or 6 signs (Covariateadjusted pooled OR 1.01, 95\% Cl 0.79-1.28). However, a positive non-significant gradient in association with the number of findings (Covariate-adjusted OR for any one clinical finding $\mathrm{OR}=0.91,95 \% \mathrm{Cl} 0.61-1.35$; any two findings $\mathrm{OR}=1.20,95 \% \mathrm{Cl} 0.84-1.73$, or 3 or more findings $\mathrm{OR}=1.31,95 \% \mathrm{Cl} 0.91-1.89$ ) was observed. Conclusions: It was found that more than half of the studied population presented a sedentary lifestyle. Even though this factor was not associated with individual signs and symptoms, a nonsignificant gradient was found, possibly related to a short exposure that may explain these results. [Villar JC, Herrera

\section{Introducción}

El sedentarismo es uno de factores de riesgo cardiovascular (FRCV) identificado desde los primeros resultados del estudio de Framingham $(1,2)$. Al igual que otros FRCV, este depende de aspectos de comportamiento, determinado a su
VM, Moreno-Medina KJ, Castellanos-Domínguez YZ, Martínez LX, Cortés OL. Can Sedentarism be Associated with Alarm Clinical Findings of Chronic Diseases in Young Adults? An Analysis in CHICAMOCHA Project. CHICAMOCHA. MedUNAB 2015; $18(1):$ 42-50]

Keywords: Motor Activity; Sedentary Lifestyle, Risk factors, Chronic Disease, Primary Prevention.

\section{Resumo}

Introdução: A associação entre sedentarismo e doenças crônicas não transmissíveis (DCNT) requer décadas de exposição. É possível que esta se manifeste mais cedo, pelo que se tem observado clinicamente em alguns adultos jovens. Objetivo: Testar a hipótese de que um estilo de vida sedentário em adultos jovens está associada com alguns sinais ou sinais de alerta para o desenvolvimento de doenças não transmissíveis. Metodologia: Usando a avaliação inicial (2000-2003) do projeto CHICAMOCHA, em 1539 doadores de sangue clinicamente saudáveis com testes de rastreio negativos (idade média de 36 anos, 8.3 anos, $66 \%$ do sexo masculino), estudou-se a associação entre sedentarismo e uma série de achados clínicos. O sedentarismo foi definido como atividade física moderadaintensa $\leq 150$ minutos/semana (incluindo trabalho). O desfecho primário foi o composto de 11 resultados (cinco sintomas e 6 sinais) de alarme encontrados na avaliação médica. A associação foi estimada utilizando um modelo de regressão logística ajustado para co-variáveis. Resultados: Verificou-se que $56.9 \%$ (IC 95\% 54.3-59.3) dos participantes eram sedentários. $\mathrm{Na}$ análise multivariada, o sedentarismo foi positivamente associado com o estado civil de solteiro e negativamente ao fato de estar empregado. Não foram encontradas associações significativas no agregado composto por 5 sintomas (OR ajustado 1,07, IC95\% 0,90-1,26), 6 sinais (OR ajustado 1.01, IC95\% $0.79-1.28)$. No entanto, é observado, um gradiente positivo, nada significativo pela descoberta presente (1 resultado OR $=0.91, \mathrm{IC} 95 \% 0.61-1.35), 2$ resultados (OR $=1.20, \mathrm{IC} 95 \%$ $0.84-1.73$ ), 3 ou mais resultados (OR $=1.31$, IC $95 \%$ 0.91-1.89). Conclusões: Mais da metade da população do estudo foi encontrada sedentária. Embora este fator não foi encontrado associado com sinais ou sintomas individualmente, foi identificado um gradiente não significativo com o número destes achados, possivelmente relacionada com o tempo de exposição relativamente curto. [Villar JC, Herrera VM, Moreno-Medina KJ, CastellanosDomínguez YZ, Martínez-Contreras LX, Cortés OL. Poderia associar-se o sedentarismo às conclusões clínicas de alarme de doenças crônicas em adultos jovens? Análise no projeto CHICAMOCHA. MedUNAB 2015; 18 (1): 42-50].

Palavras-chave: Atividade Motora; Estilo de Vida Sedentário; Fatores de Risco; Doença Crônica; Prevenção Primária.

vez por factores sociales y culturales. El sedentarismo ha sido ligado al estilo de vida impuesto por la vida moderna en grandes núcleos urbanos, en donde un mayor acceso a bienes industriales y recursos tecnológicos resulta en una menor demanda de esfuerzo físico de los individuos $(3,4)$. La adopción de un estilo de vida con tales características ha sido considerada factor predisponente no solo para el desarrollo 
de enfermedad cardiovascular, sino para diversas enfermedades crónicas no transmisibles (ECNT) para mortalidad por todas las causas (5). La OMS estimó que, para el año 2004, a través de esas asociaciones, eran atribuibles al sedentarismo el $5.5 \%$ de las muertes $(3,218,535$ decesos) a nivel global (siendo el $6.6 \%$ para países de medianos y bajos ingresos)(6). En los países como Colombia, la creciente urbanización ha sido señalada como uno de los factores potencialmente contribuyentes a la epidemia de enfermedad cardiovascular (ECV) advertida en las décadas recientes $(6,7,8,9)$. En el país se registró un aumento dramático de la población urbana, que pasó de ser del $29 \%$ en 1938 a un $78 \%$ en el censo de $2005(10,11)$. Simultáneamente, la ECV pasó a ser la primera causa de mortalidad por enfermedad en la población en las últimas décadas (12). En este contexto de la transición epidemiológica, las (ECNT) constituyen hoy el $80 \%$ de la carga de la enfermedad (13).

La Encuesta Nacional de la Situación Nutricional de Colombia (ENSIN) de 2010 estimó que 46.5\% de los adultos (edad 18-64 años) residentes en zonas urbanas colombianas eran inactivos. Solo el $19.9 \%$ cumplía con las recomendaciones de actividad física en el tiempo libre (14). En el marco de diferentes iniciativas internacionales en los años recientes, se han iniciado campañas para aumentar la conciencia sobre la importancia de la actividad física (AF) para el mantenimiento de la salud $(15,16)$. La práctica de la AF se ha relacionado inversamente con la edad $(17,18)$. Por tanto, el impacto del sedentarismo frente a diversas ECNT y calidad de vida posiblemente tenga un gradiente con el tiempo de exposición. La identificación de la magnitud del sedentarismo en adultos jóvenes, expuestos a este factor por un menor tiempo, y de su asociación con desenlaces intermedios permitiría informar mejor las recomendaciones sobre la práctica de la AF más tempranamente.

El propósito de este estudio fue probar la hipótesis de que en adultos jóvenes el sedentarismo se asocia con algunos signos o síntomas de alarma para el desarrollo posterior de ECNT.

\section{Metodología}

\section{Población del estudio}

En desarrollo del proyecto "Cardiovascular Health Investigation and Collaboration to Assess the Markers and Ouctomes of Chagas disease" (CHICAMOCHA) se realizó una encuesta sobre diferentes aspectos del estado de salud en población clínicamente saludable (donantes de sangre con pruebas de tamización a enfermedades infecciosas no reactivas) entre Mayo de 2000 y Mayo del 2003 en Bucaramanga.

El protocolo fue aprobado por el comité de ética institucional, con base en la resolución 8430 del Ministerio de Salud. Se tomó como marco muestral a los donantes voluntarios incidentes de los bancos de sangre de la ciudad que hicieron donaciones entre los años 1997 y 2000. Siguiendo el propósito de la encuesta, a partir de un registro inicial de 8,671 donantes de sangre, se tomó una muestra aleatoria de los donantes seronegativos. La muestra seleccionada fue contactada por teléfono e invitada a participar, mediante una estrategia sistemática que incluía un protocolo predefinido de contacto y reclutamiento. Quienes aceptaron participar fueron invitados a la oficina del proyecto, en donde personal de la oficina del proyecto, una enfermera o una asistente de investigación condujo una entrevista que incluía preguntas sobre la identificación, características demográficas, socio-económicas y del estilo de vida de los participantes. Inmediatamente después se realizó una valoración médica internista, incluyendo el registro de medidas antropométricas, medición de signos vitales y registro de un electrocardiograma de superficie de 12 derivaciones. Ninguna de las personas que recolectó la información conocía los propósitos específicos del estudio. Para tal efecto, el electrocardiograma fue interpretado por un médico internista en ausencia de información demográfica o clínica de los participantes.

\section{Factor de exposición y desenlaces de interés}

La exposición de interés en el estudio fue el sedentarismo, definido como la declaración de una actividad física semanal $\leq 150$ minutos/semana de actividad moderada-intensa durante su tiempo libre (19). Debido a la situación social y laboral de Colombia, se excluyó de esta definición a las personas que declararon AF con intensidad moderadaintensa por una duración $\geq 150$ minutos/semana en el trabajo. El desenlace con el cual se buscó la asociación fue un grupo de hallazgos clínicos considerados de alarma identificados a lo largo de la encuesta, cuya presencia demandaría algún tipo de intervención del equipo de salud. El desenlace primario del estudio fue el compuesto de 11 de esos hallazgos clínicos, los cuales fueron divididos en 5 indicadores subjetivos (síntomas) y 6 indicadores objetivos (signos) de alarma o pobre estado de salud. Los desenlaces secundarios fueron el compuesto de los signos y el compuesto de los síntomas de alarma.

Los síntomas de alarma fueron la auto calificación de a) un mal estado de salud; b) una mala calidad del sueño; c) insatisfacción en la vida sexual; d) percepción de alto estrés, o e) la solicitud frecuente de atención médica. Estos desenlaces fueron calificados por los participantes en un cuestionario en el que se establecían 5 opciones que daban una calificación gradual al síntoma en cuestión. Se consideró presente el síntoma, cuando el participante lo calificaba dentro de las tres categorías inferiores (V.g. calificar la calidad del sueño como regular, mala o pésima). Para evaluar el estrés se preguntó separadamente a los participantes sobre estrés en el trabajo, en el hogar y estrés financiero. Se consideró alto estrés cuando el participante calificó su percepción de estrés como "mayor al esperado", "alto" o "exagerado" en al menos dos de los tres ambientes. Una 
solicitud frecuente de consulta médica se consideró cuando el participante había solicitado más de dos citas médicas ambulatorias no consideradas controles periódicos de salud o había concurrido más de una vez a un servicio de urgencias en los últimos dos años.

Los 6 signos de alarma derivados de la valoración médica fueron: a) Presión arterial sistólica mayor a $130 \mathrm{~mm} \mathrm{de} \mathrm{Hg}$, tomando la media de una medición en cada brazo; b) Índice de masa corporal mayor a 30\%; c) Índice cintura-cadera mayor a 1en hombres o 0.9 en mujeres; d) Glucemia $\geq 110$ $\mathrm{mg} / \mathrm{dl}$; e) Frecuencia cardiaca mayor a 75 latidos por minuto, derivada del intervalo R-R de un electrocardiograma tomado al final de la valoración médica; f) Electrocardiograma considerado anormal, por la presencia de algún trastorno definido del ritmo o la conducción (bloqueo aurículoventricular, bloqueos de rama, extrasístoles ventriculares) o signos de hipertrofia ventricular izquierda.

\section{Análisis estadístico}

Para determinar preliminarmente su asociación, se condujo un análisis univariado del conteo (para las variables categóricas) o la media (para las variables continuas) de las variables de interés de acuerdo con la presencia o no de sedentarismo. Para cada factor entre los participantes considerados sedentarios $(\operatorname{Sed}[+])$ o no sedentarios $(\operatorname{Sed}[-])$ se calcularon los valores de $\mathrm{p}$ de acuerdo a la prueba de Chi cuadrado o T de student. Los factores de interés en los cuales se encontrara una asociación definitiva o potencial ( $\mathrm{p} \square 0.15$ ) se consideraron elegibles para ser incluidos en análisis posteriores de asociación como potenciales factores de confusión para la asociación entre el sedentarismo y los desenlaces del estudio.

La estimación final de la asociación objeto del estudio se realizó mediante el cálculo de Odds Ratios (OR) en un modelo de regresión logística multivariado en el que se incluyeron estos factores de confusión. Para la selección de estos factores, se examinaron diversos modelos incluyendo los diferentes factores elegibles o sus interacciones en orden descendente de asociación, utilizando la técnica de regresión paso a paso. Para ser incluidos en el modelo, este debía incluir factores cuya asociación independiente de otros cofactores rindiera un valor de $\mathrm{p} \square 0.20$ para cada uno de ellos. Por su relación conocida con los desenlaces del estudio, se consideró la entrada forzosa del tabaquismo a la lista de covariables incluidas en el modelo seleccionado.

Usando este modelo de regresión logística se calcularon ORs para la asociación entre cada uno de los 11 desenlaces de interés y el sedentarismo. Se calculó también un estimado compuesto para los indicadores subjetivos, otro para los indicadores objetivos y un gran estimado compuesto general para la asociación entre todos los signos clínicos de alarma y el sedentarismo. Finalmente, se determinó la asociación en la forma de dosis-efecto, a partir del número de indicadores presentes en cada individuo. Tomando como categoría de referencia a la ausencia de indicadores de alarma, se calcularon ORs para la asociación entre la presencia de uno, dos o tres o más indicadores subjetivos u objetivos, al igual que para la presencia de uno, dos, tres o más de cualquiera de estos indicadores y el sedentarismo. Usando la técnica de regresión paso a paso se incluyeron o excluyeron factores en orden descendente de acuerdo con la jerarquía de su asociación.

\section{Resultados}

\section{Características socio-demográficas de los participantes}

De acuerdo con el muestreo establecido para la encuesta, se seleccionaron 1645 participantes que fueron contactados telefónicamente y asistieron a la valoración. Todos los asistentes aceptaron por escrito participar luego de ser informados verbalmente acerca de la encuesta. En 106 de estos participantes no hubo un registro de información que permitiera clasificarlos o no como sedentarios, de acuerdo con nuestra definición operativa.

Del total de participantes, 875 (56.9\%, IC95\% 54.3\% $59.3 \%$ ) se encontraron sedentarios. En el análisis univariado (que se resume en la Tabla 1), los participantes Sed[+] eran

Tabla 1. Distribución de las características socio-demográficas de la población de estudio según la presencia o no de sedentarismo.

\begin{tabular}{lccc}
\hline \multicolumn{1}{c}{ Factor } & $\begin{array}{c}\text { Sedentarismo }[+] \\
\mathrm{n}=875\end{array}$ & $\begin{array}{c}\text { Sedentarismo } \\
{[-]} \\
\mathrm{n}=664\end{array}$ & Valor de $\mathrm{p}$ \\
\hline Edad Promedio (DS) & $36.1(8.6)$ & $35.4(8.1)$ & 0.089 \\
\hline$\geq 40$ años $\mathrm{n}(\%)$ & $324(37.0)$ & $210(31.6)$ & 0.029 \\
\hline Género, Masculino $\mathrm{n}(\%)$ & $522(59.7)$ & $467(70.3)$ & $<0.001$ \\
\hline Afiliación al SGSS ${ }^{*}$ Contributivo $\mathrm{n}(\%)$ & $593(67.8)$ & $430(64.8)$ & 0.242 \\
\hline Estrato Socioeconómico 1-3 $\mathrm{n}(\%)$ & $623(71.2)$ & $464(69.9)$ & 0.489 \\
\hline Estado civil, Soltero $\mathrm{n}(\%)$ & $256(29.3)$ & $185(27.9)$ & 0.027 \\
\hline Educación básica $\mathrm{n}(\%)$ & $546(62.4)$ & $404(60.8)$ & 0.461 \\
\hline Ocupación Empleado/independiente $\mathrm{n}(\%)$ & $597(68.2)$ & $545(82.1)$ & $<0.001$ \\
\hline
\end{tabular}

* Sistema General de Seguridad Social 
Tabla 2. Distribución de algunos factores de percepción del estado de salud de la población de estudio según la presencia o no de sedentarismo.

\begin{tabular}{lccc}
\hline \multicolumn{1}{c}{ Factor } & $\begin{array}{c}\text { Sedentarismo }[+] \\
\mathbf{n}=875\end{array}$ & $\begin{array}{c}\text { Sedentarismo [-] } \\
\mathbf{n}=664\end{array}$ & Valor de $\mathbf{p}$ \\
\hline Percepción de altos niveles de estrés $\mathrm{n}(\%)$ & $169(19.3)$ & $153(23.0)$ & 0.075 \\
\hline Percepción de mal estado de salud $\mathrm{n}(\%)$ & $68(7.8)$ & $71(10.7)$ & 0.053 \\
\hline Percepción de mala calidad del sueño $\mathrm{n}(\%)$ & $256(29.3)$ & $156(23.5)$ & 0.013 \\
\hline Insatisfacción vida sexual $\mathrm{n}(\%)$ & $96(11.0)$ & $75(11.3)$ & 0.956 \\
\hline Solicitud frecuente cita(s) médica(s) $\mathrm{n}(\%)$ & $250(28.6)$ & $151(22.7)$ & 0.010 \\
\hline
\end{tabular}

Tabla 3. Distribución de signos clínicos de riesgo cardiovascular en la población de estudio según la presencia o no de sedentarismo.

\begin{tabular}{lccc}
\hline \multicolumn{1}{c}{ Factor } & $\begin{array}{c}\text { Sedentarismo }[+] \\
\mathrm{n}=875\end{array}$ & $\begin{array}{c}\text { Sedentarismo }[-] \\
\mathrm{n}=664\end{array}$ & $\begin{array}{c}\text { Valor de } \\
\mathrm{p}\end{array}$ \\
\hline Frecuencia cardíaca $(\mathrm{Ipm})$ Promedio (DS) & $65.2(8.6)$ & $64.4(9.0)$ & 0.074 \\
Frecuencia cardiaca $\geq 75 \mathrm{n}(\%)$ & $119(13.6)$ & $80(12.0)$ & 0.352 \\
\hline Presión arterial sistólica $(\mathbf{m m ~ H g ) ~ P r o m e d i o ~ ( D S ) ~}$ & $117.1(12.9)$ & $117.5(11.7)$ & 0.532 \\
Presión arterial sistólica $\geq 130 \mathrm{n}(\%)$ & $120(13.7)$ & $92(13.9)$ & 0.960 \\
\hline Índice de masa corporal Promedio (DS) & $25.8(3.9)$ & $25.6(4.2)$ & 0.260 \\
Índice de masa corporal $\geq 30 \mathrm{n}(\%)$ & $118(13.5)$ & $81(12.2)$ & 0.407 \\
\hline Índice cintura/cadera Promedio (DS) & $0.87(0.09)$ & $0.88(0.08)$ & 0.399 \\
$\geq 1$ Hombres $\geq \mathbf{0 . 9}$ mujeres $\mathrm{n}(\%)$ & $102(11.7)$ & $71(10.7)$ & 0.515 \\
\hline Glicemia Promedio (DS) & $81.7(17.1)$ & $81.9(19.7)$ & 0.835 \\
Glicemia $\geq 110 \mathrm{n}(\%)$ & $39(4.5)$ & $32(4.8)$ & 0.732 \\
\hline Electrocardiograma anormal $\mathrm{n}(\%)$ & $99(11.3)$ & $91(13.7)$ & 0.160 \\
\hline
\end{tabular}

Tabla 4. Modelo multivariado de regresión logística. Factores asociados a sedentarismo. Parámetros del modelo: Hosmer-Lemeshow=99.7\%; R2=91\%; $\mathrm{AUC}=68 \%$. Se incluyeron al modelo variables con valor $p<0.10$ en el análisis bivariado.

\begin{tabular}{lcccc}
\hline \multirow{2}{*}{ Variables } & \multirow{2}{*}{ OR } & \multicolumn{2}{c}{ IC95\% } & \multirow{2}{*}{ Valor $\mathbf{p}$} \\
\cline { 3 - 4 } & & LI & LS & \\
\hline Estado civil soltero & 1.65 & 1.15 & 2.36 & 0.006 \\
\hline Ocupación empleado/independiente & 0.30 & 0.14 & 0.61 & 0.001 \\
\hline
\end{tabular}

predominantemente de sexo femenino $(p<0.001)$, no empleados $(\mathrm{p}<0.001)$ más frecuentemente mayores de 40 años $(\mathrm{p}=0.029)$ y solteros $(\mathrm{p}=0.027)$. No se encontraron diferencias por la presencia de sedentarismo respecto a los años de educación recibidos, estrato socioeconómico de la residencia o tipo de régimen de afiliación al sistema de salud.

\section{Relación entre el sedentarismo y los desenlaces del estudio}

El análisis univariado de los síntomas de alarma en relación al sedentarismo se resume en la Tabla 2. Una menor proporción de participantes sedentarios declararon vivir con altos niveles de estrés ( $19.3 \%$ versus $23.0 \%$ en Sed[-], $\mathrm{p}=0.075)$, o percibían tener un mal estado de salud $(7.8 \%$ versus 10.7 en $\operatorname{Sed}[-], p=0.053)$. Por el contrario, la percepción de mala calidad del sueño $(29.3 \%$ versus $23.5 \%$ en Sed[-], $\mathrm{p}=0.013$ ) y la solicitud frecuente de citas médicas $(28.6 \%$ versus $22.7 \%$ en $\operatorname{Sed}[-], p=0.010)$ fue más frecuentemente reportada por las personas identificadas como sedentarias. El reporte de insatisfacción en la vida sexual fue muy similar en ambos grupos (11.0\% y $11.3 \%$, respectivamente, $\mathrm{p}=0.956$ ). El mismo tipo de análisis para los signos de alarma (Tabla 3) no mostró diferencias significativas $(\mathrm{p}<0.05)$ de acuerdo con la presencia o no de sedentarismo en los participantes. Se encontró una tendencia consistente en los niveles de estas variables, o la frecuencia por encima de los puntos de corte establecidos para los grupos de referencia.

\section{Análisis multivariado}

El modelo incluyó las covariables descritas en la Tabla 1 potencialmente asociadas al sedentarismo. Este reveló una asociación positiva con el sedentarismo para el estado civil soltero $(\mathrm{OR}=1.65$, IC95\% $1.15-2.36)$ y negativa $(\mathrm{OR}=$ 0.30 IC95\% 0.14 - 0.61) para estar empleado. Estas asociaciones y el diagnóstico del modelo se muestran en la Tabla 4. 
Tabla 5. Resumen de los estimados de la asociación entre los desenlaces del estudio y el sedentarismo. Los estimados son ajustados por variaciones de edad, género, estrato social, estado civil, ocupación y nivel educación. Los ítems relacionados con una auto-percepción de mala salud o calidad de vida (parte superior) y los signos de alarma en el examen clínico (parte inferior) se presentan individualmente y como un estimado compuesto. OR: Odds ratio. IC $95 \%$ : Intervalos de confianza del $95 \%$.

\begin{tabular}{lcccc}
\hline \multicolumn{1}{c}{ Variables } & $\begin{array}{c}\text { OR } \\
\text { crudo }\end{array}$ & IC95\% & $\begin{array}{c}\text { OR } \\
\text { ajustado }\end{array}$ & IC95\% \\
\hline Indicadores subjetivos (síntomas) de alarma & & & & \\
\hline Percepción de altos niveles de estrés & 0.79 & $0.61-1.02$ & 0.91 & $0.59-1.39$ \\
\hline Percepción de mal estado de salud & 0.71 & $0.50-1.00$ & 0.48 & $0.22-1.02$ \\
\hline Percepción de mala calidad del sueño & 1.34 & $1.06-1.69$ & 1.23 & $0.83-1.82$ \\
\hline Insatisfacción vida sexual & 1.01 & $0.73-1.39$ & 1.15 & $0.66-2.01$ \\
\hline Solicitud frecuente cita(s) médica(s) & 1.35 & $1.07-1.71$ & 1.47 & $0.97-2.21$ \\
\hline Estimado compuesto & 1.03 & $\mathbf{0 . 9 4 - 1 . 1 4}$ & 1.07 & $\mathbf{0 . 9 0 - 1 . 2 6}$ \\
\hline Indicadores objetivos (signos) de alarma & & & & \\
\hline Frecuencia cardiaca $\geq 75$ & 1.15 & $0.85-1.56$ & 1.34 & $0.78-2.32$ \\
\hline Presión arterial sistólica $\geq 130$ & 0.99 & $0.74-1.33$ & 1.09 & $0.61-1.94$ \\
\hline Índice de masa corporal $\geq 30$ & 1.13 & $0.83-1.54$ & 1.30 & $0.72-2.33$ \\
\hline Índice cintura/cadera Masc $\geq 1$ Fem $\geq 0.9$ & 1.11 & $0.80-1.53$ & 0.50 & $0.27-0.90$ \\
\hline Glicemia $\geq 110$ & 0.92 & $0.57-1.48$ & 1.06 & $0.45-2.49$ \\
\hline Electrocardiograma anormal & 0.80 & $0.59-1.09$ & 1.03 & $0.59-1.79$ \\
\hline Estimado compuesto & 1.02 & $\mathbf{0 . 8 9 - 1 . 1 6}$ & $\mathbf{1 . 0 1}$ & $\mathbf{0 . 7 9 - 1 . 2 8}$ \\
\hline
\end{tabular}

Establecidas las covariables a incluir en el modelo de asociación de los desenlaces de interés, se calcularon ORs para asociar sedentarismo con los indicadores clínicos de alarma. La Tabla 5 resume estas asociaciones. Para los síntomas de alarma, no se encontró ninguna asociación significativa individual. La dirección de estas asociaciones tampoco fue consistente. En forma agregada, se encontró una asociación positiva, no significativa $(\mathrm{OR}=1.07$, IC 95\% 0.90-1.26). Para los signos de alarma, se encontró una asociación negativa con tener el índice cintura-cadera por encima del nivel de corte (1 para hombres, 0.9 para mujeres). Sin embargo, la dirección de la asociación fue la opuesta para 5 de los 6 signos examinados. El estimado agregado de asociación fue neutro (OR=1.01, IC95\% 0.79-1.28).

\section{Gradiente dosis-respuesta en la asociación}

La exploración de esta hipótesis mostró un gradiente positivo, aunque no significativo por número de signos presentes, o el agregado de síntomas y signos (Tabla 6).

Tabla 6. Gradiente de la asociación progresiva entre el acumulado de indicadores y el sedentarismo. Los estimados son ajustados por variaciones de edad, género, estrato social, estado civil, ocupación y nivel educación. Se presenta el acumulado de indicadores subjetivos (arriba), objetivos (en el medio) o la suma de todos los indicadores (abajo). El grupo sin indicadores presentes es el nivel de referencia usado para la comparación en cada parte de la figura. OR: Odds ratio. IC 95\%: Intervalos de confianza del 95\%.

\begin{tabular}{|c|c|c|c|c|c|}
\hline Variables & & $\begin{array}{c}\text { OR } \\
\text { crudo }\end{array}$ & $\mathrm{IC} 95 \%$ & $\begin{array}{c}\text { OR } \\
\text { ajustado }\end{array}$ & IC95\% \\
\hline \multicolumn{6}{|c|}{ Número de síntomas de alarma presentes } \\
\hline & 1 & 0.96 & $0.78-1.18$ & 1.06 & $0.75-1.51$ \\
\hline & 2 & 1.01 & $0.82-1.25$ & 1.20 & $0.84-1.73$ \\
\hline & w & 1.10 & $0.83-1.47$ & 1.09 & $0.66-1.81$ \\
\hline \multicolumn{6}{|c|}{ Número de signos de alarma presentes } \\
\hline & 1 & 0.92 & $0.74-1.15$ & 0.99 & $0.67-1.47$ \\
\hline & 2 & 0.95 & $0.7-1.31$ & 1.17 & $0.66-2.08$ \\
\hline & $\geq 3$ & 1.04 & $0.63-1.70$ & 1.22 & $0.41-3.60$ \\
\hline \multicolumn{6}{|c|}{$\begin{array}{l}\text { Número de síntomas o signos de alarma } \\
\text { presentes }\end{array}$} \\
\hline & 1 & 1.00 & $0.79-1.26$ & 0.91 & $0.61-1.35$ \\
\hline & 2 & 1.16 & $0.93-1.44$ & 1.20 & $0.84-1.73$ \\
\hline & $\geq 3$ & 1.19 & $0.96-1.47$ & 1.31 & $0.91-1.89$ \\
\hline
\end{tabular}


Mientras que para la presencia de un signo hubo un estimador de asociación neutro ( $\mathrm{OR}=0.99$, IC95\% 0.67 1.47), hubo una asociación creciente para la presencia de 2 $(\mathrm{OR}=1.17$, IC95\% 0.66-2.08) o 3 o más signos $(\mathrm{OR}=1.22$, IC95\%0.41, 3.60). En el mismo sentido, la asociación para el agregado de signos-síntomas partió de 0.91 (IC95\% 0.61 $-1.35)$ para la presencia de un rasgo, incrementando a 1.20 (IC95\% 0.84-1.73) para 1 de esos rasgos y 1.31 (IC95\% $0.91-1.89$ ) para la presencia de 3 o más de estos rasgos.

\section{Discusión}

\section{Principales hallazgos}

En este estudio se encontró que, en los donantes de sangre en una ciudad como Bucaramanga, para los primeros años del siglo, la prevalencia de sedentarismo era del $57 \%$.

Este factor de riesgo se distribuye con mayor frecuencia en personas solteras y con menor frecuencia en empleados, quizá debido a la preeminencia del trabajo con poca mecanización en el país. Fue por esta razón que decidimos incluir, además de la AF en el tiempo libre, la AF en el trabajo para nuestra definición operativa de sedentarismo. Este hallazgo resalta la necesidad de particularizar las definiciones de estilo de vida sedentario y las intervenciones para promover la AF con base en las características socioeconómicas y culturales de cada sociedad(20,21).

No se encontró una asociación positiva, ni una dirección consistente entre el sedentarismo y los diferentes hallazgos clínicos de alarma seleccionados. Sin embargo, en este estudio se identificó un gradiente positivo en la asociación con la presencia de múltiples de estos signos o síntomas. La AF ha sido extensamente relacionada en otros estudios con varias ECNT o sus factores de riesgo (17,22,23), o con hallazgos clínicos relacionados, evaluados aquí, tales como síntomas depresivos (24), dolor (25), medidas de calidad de vida relacionada con la salud (26), la percepción del estado de salud, en residentes de países como Brasil (27), China (28), Corea (29), o España (30); con una mayor demanda de cuidado de salud(31), una mala calidad del sueño(22), o con los signos de alarma de tipo metabólico y antropométrico (32) . Dado que nuestro propósito fue explorar estas relaciones en adultos jóvenes (edad promedio 36 años), es posible que no hubiese un suficiente tiempo de exposición para identificar esta asociación con la suficiente fortaleza. Es posible que en el seguimiento actual de la cohorte, con una década más de exposición, estas relaciones se puedan establecer más claramente.

\section{Fortalezas y limitaciones}

Entre las fortalezas de este estudio cabe destacar el carácter prospectivo de su diseño y de la recolección de información en un escenario geográfico e histórico en donde se tiene relativamente poca información. La evaluación de línea de base de CHICAMOCHA permitió la recolección de un amplio y variado número de desenlaces de salud. Estos incluyen algunos de tipo comportamental (subjetivos), así como métricas potencialmente relacionadas con el sedentarismo. Adicionalmente, el tamaño de la muestra en esta encuesta permite una adecuada estimación de la magnitud del sedentarismo y de sus factores sociodemográficos asociados. Por esta razón consideramos poco probable que una asociación de magnitud clínicamente importante no hubiese sido identificada. Finalmente, en este estudio evaluamos los desenlaces individuales, como en forma agregada, lo que permitió un triple examen de la fortaleza de la asociación en magnitud, consistencia y gradiente del número de hallazgos.

También este estudio tiene varias limitaciones. La más importante deriva de la población evaluada, los donantes de sangre. Nuestros resultados aplican a la población general urbana en la medida que los donantes de los bancos de sangre pueden representarla. Por su misma naturaleza (derivada del propósito primario del estudio, servir de control a donantes seropositivos a Trypanosoma cruzi para hacer inferencias de diagnóstico temprano de cardiomiopatía de Chagas), el nivel de salud de esta población podría representar un sobreestimado (33). En cuanto a la validez interna del estudio, tiene dos implicaciones: En primer lugar, es posible que la prevalencia de sedentarismo estuviera sobreestimada (respecto a la población general). Sin embargo la ENSIN, una encuesta en población general en adultos de hasta 64 años, arrojó un dato de sedentarismo inferior a la de CHICAMOCHA. Estos dos estudios también utilizaron métodos diferentes de medición. ENSIN utilizó dos dominios (tiempo libre y transporte) del International Physical Activity Questionnaire (IPAQ). CHICAMOCHA (cuyos participantes tenían hasta 56 años en la línea de base) en cambio registró información sobre la duración e intensidad de la AF en el tiempo libre y en el trabajo (lo que podría o no incluir el transporte según la interpretación del participante). Para el momento del diseño de ENSIN (estudio posterior a CHICAMOCHA), sus investigadores habían decidido excluir el módulo de AF en el trabajo de IPAQ (basados en experiencia de su aplicación en Brasil y Colombia) por considerar que podría generar sobreestimados (34). En tanto, otro informe sobre AF en población adulta en Bogotá en el 2003 la prevalencia de sedentarismo fue $63.2 \%$ en 3000 adultos de 18 a 65 años residentes en estratos 1-4, basado en la versión corta del IPAQ (35). Como no es posible comparar los niveles de AF en donantes de sangre y población general con métodos similares, la magnitud reportada aquí cabría dentro de un rango con similares implicaciones en salud pública.

En segundo lugar, el tipo de población estudiada reduce las posibilidades de identificar asociaciones con hallazgos clínicos de alarma. Sin embargo, dado que estas son expresadas en términos relativos (a sus pares menos activos físicamente) y no absolutos, el sesgo del "voluntario saludable" tendría un menor impacto sobre los resultados 
encontrados. Tampoco la falta de precisión estadística, pues $58 \%$ de la población tenía la exposición de interés y el número de absoluto para cada desenlace era mayor a 200 en 5 de los 11 desenlaces y superaba los 100 en todos, excepto un desenlace (Glucemia $\geq 110 \mathrm{mg} / \mathrm{dl}$ con 71 eventos). Finalmente, en cuanto a la validez externa, habría definitivamente limitaciones en la extrapolación de estos hallazgos, dado el carácter "negativo" de nuestros resultados". En este caso, no podría descartarse la existencia de las asociaciones de interés de haber estudiado otro tipo de población.

\section{Implicaciones}

Respecto a la práctica clínica, a pesar de una conciencia generalizada sobre la AF para el mantenimiento de la salud, las recomendaciones sobre el inicio y mantenimiento de la AF tienen como población objeto primordialmente los adultos, especialmente por la prevención de la diabetes(36). Con una menor fortaleza, esta recomendación se extiende a otros grupos etarios. En una forma similar, las recomendaciones sobre el consumo de sal tienen mayor énfasis en pacientes con hipertensión arterial, que como instrumento para la prevención de la hipertensión arterial, a pesar de haber identificado un continuo de esta relación en un rango amplio de población $(37,38)$.

El sedentarismo es el factor de riesgo cardiovascular más prevalente entre los factores convencionales, y quizá un factor aglutinador y potenciador de enfermedad cardiovascular y otras ECNT. Bajo esas consideraciones, el manejo del sedentarismo requeriría de una estrategia agresiva y urgente que por su dependencia con la organización de la sociedad actual tendría que tener un carácter más colectivo que individual. Por tanto, aquí la participación de los gobiernos se hace mucho más trascendente, tal como ha sido previamente documentado (39). La implementación de las medidas con mayor costoefectividad y el diseño de la futura investigación en el área requeriría un abordaje "ecológico"(40), que permita probar nuevas y creativas intervenciones conducentes a la reversión de la tendencia a la adopción de estilos de vida no saludables, contando con la voluntad de acción de los gobernantes y la población.

\section{Conclusiones}

Este estudio presenta un abordaje a la cuantificación del sedentarismo en la ciudad colombiana y a la asociación con desenlaces intermedios de salud (11 hallazgos clínicos de alarma, 5 síntomas y 6 signos) en adultos jóvenes. El 58\% de esta población se encontró sedentaria. No se identificaron relaciones entre el sedentarismo y los desenlaces evaluados en forma individual, pero se observó un gradiente positivo con el número de hallazgos en un individuo dado. Es posible que esta falta de asociación se deba a un insuficiente tiempo de exposición, o en menor medida al carácter de voluntarios sanos inherente a los donantes de sangre como población de estudio.

\section{Conflicto de intereses}

Los autores declaran no tener conflicto de interés.

\section{Agradecimientos}

Este estudio ha sido posible gracias a la financiación del fondo de ayudas para la comunidad de ECOPETROL y COLCIENCIAS contrato 172-2002.

\section{References}

1. Kannel WB, Wilson P BS. Epidemiological assessment of the role of physical activity and fitness in development of cardiovascular disease. Am Hear J. 1985;109(4): 876-85.

2. Kannel WSP. Some health benefits of physical activity. The Framingham Study. Arch Intern MeD. 1979;139(8):857-61.

3. RR P. Urbanization as a determinant of health: a socioepidemiological perspective. Soc Work Public Heal. 2014;29(4):335-41.

4. Jebb SA MM. Contribution of a sedentary lifestyle and inactivity to the etiology of overweight and obesity: current evidence and research issues. Med Sci Sport Exerc. 1999;31(S11):S534-41.

5. Oggioni C, Cena H, Wells JC, Lara J, Celis-Morales C SM. Association between worldwide dietary and lifestyle patterns with total cholesterol concentrations and DALYs for infectious andw cardiovascular diseases: An ecological analysis. J Epidemiol Glob Heal. 2015;

6. Organization WH. Global Health Risks: Mortality and burden of disease attributable to selected major risks [Internet]. 2009. Available from: http://www.who.int/ healthinfo/global_burden_disease/GlobalHealthRisks_r eport_full.pdf?ua $=1 \& u a=1$

7. Blackmore HL OS. Programming of cardiovascular disease across the life-course. J Mol Cell Cardiol. 2015;83:122-30.

8. Reddy KS YS. Emerging epidemic of cardiovascular disease in developing countries. Circulation. 1998;97(6):596-601.

9. Anand SS, Yusuf S, Vuksan V, Devanesen S, Teo KK, Montague PA, Kelemen L, Yi C, Lonn E, Gerstein H, Hegele RA MM. Differences in risk factors, atherosclerosis, and cardiovascular disease between ethnic groups in Canada: the Study of Health Assessment and Risk in Ethnic groups (SHARE). Lancet. 2000;356(9226):279-84.

10. DANE. Censo 2005. Disponible en www.dane.gov.co. Consultado el 22 de junio 2015.

11. DANE. Censo General 2005. Departamento Administrativo Nacional de Estadística, Colombia [Internet]. 2015. Available from: http://www.dane.gov.co/ index.php/poblacion-y-demografia/censos 
12. Ministerio de salud y Protección. Análisis de situación en Salud según regiones Colombia. 2013.

13. Peñaloza RE, Salamanca N, Rodriguez JM, Rodriguez J BA. Estimación de la carga de enfermedad para Colombia, 2010. 2014.

14. Fonseca Z, Heredia AP, Ocampo PR, Forero $Y$, Sarmiento OL AM et al. Encuesta Nacional de la Situación Nutricional en Colombia (ENSIN) 2010 [Internet]. Instituto Colombiano de Bienestar Familiar. 2015 . A v a i a b l e f r o m: http://www.icbf.gov.co/portal/page/portal/PortallCBF/Bi enestar/ENSIN1/ENSIN2010/LibroENSIN2010.pdf

15. (IDRD) ID de R y D. Actividad física muévete Bogotá [ Internet]. A vailable from : www.idrd.gov.co/sitio/idrd/?q=node/467

16. Ministerio de Salud y Protección Social (MSPS). Actividad física [Internet]. Actividad Física. 2015. Available from: www.minsalud.gov.co/salud/ Paginas/Actividad-F\%C3\%ADsica.aspx

17. Martins TG, Assis MA, Nahas MV, Gauche H ME. Leisure-time physical inactivity in adults and factors associated. Rev Saude Publica. 2009;43(5):814-24.

18. (CDC) $C$ for $D C$ and P. Vital signs: walking among adults--United States, 2005 and 2010. MMWR Morb Mortal Wkly Rep. 2012;61(31):595-601.

19. Organization WH. Recomendaciones Mundiales sobre Actividad Física para la Salud. Geneva WHO Libr Cat [Internet]. 2010;1-58. Available from: http://scholar.google.com/scholar?hl=en\&btnG=Search $\& q=$ intitle:Recomendaciones+Mundiales+sobre+activid ad+F?sica+para+la+salud\#4lnhttp://whqlibdoc.who.int/ publications/2010/9789243599977_spa.pdf

20. Seefeldt V, Malina RM CM. Factors affecting levels of physical activity in adults. Sport Med. 2002;13(5):143-68.

21. Tudor-Locke C, Henderson KA, Wilcox S, Cooper RS, Durstine JL AB. In their own voices: definitions and interpretations of physical activity. Womens Heal Issues. 2003;13(5):194-9.

22. Wheaton AG, Perry GS, Chapman DP, McKnight-Eily LR, Presley-Cantrell LR CJ. Relationship between body mass index and perceived insufficient sleep among U.S. adults: an analysis of 2008 BRFSS data. BMC Public Health. 2011;11(295).

23. Pronk NP, Lowry M, Kottke TE, Austin E, Gallagher J KA. The association between optimal lifestyle adherence and short-term incidence of chronic conditions among employees. Popul Heal Manag. 2010;13(6):289-95.

24. Arredondo EM, Mendelson T, Elder JP, Marshall SJ, La FL AG. The relation of medical conditions to depressive symptoms among Latinos: leisure time physical activity as a mediator. J Heal Psychol. 2012;17(5):742-52.

25. Nilsen TI, Holtermann A MP. Physical exercise, body mass index, and risk of chronic pain in the low back and neck/shoulders: longitudinal data from the NordTrondelag Health Study. Am J Epidemiol. 2011;174(3):267-73.

26. Bize R PR. The relationship between a short measure of health status and physical activity in a workplace population. Psychol Heal Med. 2009;14(1):53-61.

27. Barros MB, Zanchetta LM, Moura EC MD. Self-rated health and associated factors, Brazil, 2006. Rev Saude Publica. 2009;43(2):27-37.
28. Lv J, Liu Q, Ren Y, Gong T, Wang S LL. Sociodemographic association of multiple modifiable lifestyle risk factors and their clustering in a representative urban population of adults: a cross-sectional study in Hangzhou, China. Int J Behav Nutr Phys Act. 2011;8(40).

29. Han MA, Kim KS, Park J, Kang MG RS. Association between levels of physical activity and poor self-rated health in Korean adults: The Third Korea National Health and Nutrition Examination Survey (KNHANES), 2005. Public Health. 2009;123(10):665-9.

30. Cimarras-Otal C, Calderon-Larranaga A, Poblador-Plou B, Gonzalez-Rubio F, Gimeno-Feliu LA A-SJ et al. Association between physical activity, multimorbidity, self-rated health and functional limitation in the Spanish population. BMC Public Health. 2014;14(1170).

31. Katz AS PN. The relationship between physical activity and care-seeking behavior among employed adults. J Phys Act Heal. 2014;11(2):313-9.

32. Bryan SN KP. The association between meeting physical activity guidelines and chronic diseases among Canadian adults. J Phys Act Heal. 2011;8(1):10-7.

33. Ullum H, Rostgaard K, Kamper-Jorgensen M, Reilly M, Melbye M NO et al. Blood donation and blood donor mortality after adjustment for a healthy donor effect. Transfusion. 2015;

34. Hallal PC, Gómez LF, Parra DC, Lobelo F, Mosquera J FA et al. Lessons learned after 10 years of IPAQ use in Brazil and Colombia. J Phys Act Heal. 2010;7(2):S259-64.

35. Gomez LF, Duperly J, Lucumi DI, Gamez R VA. Nivel de actividad física global en la población adulta de Bogotá (Colombia). Prevalencia y factores asociados. Gac Sanit. 2005;19(3):206-13.

36. Knowler WC, Barrett-Connor E, Fowler SE, Hamman RF, Lachin JM WE et al. Reduction in the incidence of type 2 diabetes with lifestyle intervention or metformin. N Engl J Med. 2002;346(6):393-403.

37. Brown IJ, Dyer AR, Chan Q, Cogswell ME, Ueshima H SJ et al. Estimating 24-hour urinary sodium excretion from casual urinary sodium concentrations in Western populations: the INTERSALT study. Am J Epidemiol. 2013;177(11):1180-92.

38. O'Donnell M, Mente A, Rangarajan S, McQueen MJ, Wang $X \mathrm{LL}$ et al. Urinary sodium and potassium excretion, mortality, and cardiovascular events. N Engl J Med. 2014;371(7):612-23.

39. Mendis S CO. The global burden of cardiovascular diseases: a challenge to improve. Curr Cardiol Rep. 2014;16(5):486.

40. Kelsey J, Whittemore A, Evans AS TW. Cross sectional and other type of studies. Methods in observational epidemiology. Oxford Uni. Oxford; 1996. 